\title{
A Novel Robust Adaptive Beamformer using Diagonal Loading
}

\author{
T.S.Jeyali Laseetha \\ Professor, Department of Electronics and \\ Communication Engineering \\ HolyCross Engineering College, Anna University \\ of Technology, Tirunelveli, \\ Tamil Nadu, INDIA
}

\author{
Dr. R.Sukanesh \\ Professor, Department of Electronics and \\ Communication Engineering \\ Thiagarajar College of Engineering, Madurai \\ Tamil Nadu, INDIA
}

\begin{abstract}
Adaptive beamfomer which utilizes MVDR beamformer along with SMI (sample matrix inversion), actual data is not available to calculate the covariance matrix. Instead, covariance matrix is estimated from the available data. It may result in bad conditioning. To avoid this, diagonal elements are introduced in the correlation matrix, which is called diagonal loading. Diagonal loading can be inserted by adding a scaled version of identity matrix to impart Robustness to the adaptive beamformer. This proves to be efficient against signal mismatch due to low sample support and helps to achieve desired sidelobe level and SINR improvement. A novel hybrid algorithm for MVDR-SMI beamformer with colored adaptive diagonal loading is proposed in this paper. The performance of the proposed method is compared with other methods such as Conventional, MVDR-SMI-Diagonal Loading, MVDR-SMIColored -DL, MVDR-SMI-Adaptive DL by conducting simulation experiments to prove its effectiveness in improving the directivity and SINR
\end{abstract}

Keywords: Smart antennas, Adaptive beamforming, Uniform Linear Array, Minimum Variance Distortionless Response Beamformer (MVDR), Sample-Matrix Inversion(SMI), Adaptive colored diagonal loading

\section{INTRODUCTION}

Adaptive beamforming reveals to be a complementary means for signal-to-interference-plus-noise-ratio (SINR) optimization [6, $7,10]$. In this paper, at antenna array elements level, the formation of a lobe structure that results from the dynamic variation of an element-space processing weight vector is controlled by an adaptive algorithm, which is the MVDRSample Matrix Inversion algorithm [2, 7, 10]. It minimizes cost function reduction of a link's SINR by ideally directing beams toward the signal-of-interest (SOI) and nulls in the directions of interference. In optimum beamformers optimality can be achieved in theory if perfect knowledge of the second order statistics of the interference is available. It involves calculation of interference plus noise correlation matrix $R_{i+n}$. For real world scenarios, the adaptive methods are followed to obtain optimality. In adaptive beamformer, the correlation matrix is estimated from collected data. In sample matrix Inversion technique a block of data is used to estimate adaptive beamforming weight vector. The estimate $\hat{R}_{i+n}$ is not really a substitute for true correlation matrix $R_{i+n}$. Hence there is degradation in performance. The SINR which is a measure of performance of the beamformer degrades as sample support (the number of data) is low. The lower band on sidelobe levels of the beamformer when no interference sources are found at an angle is also calculated. Training issues like the presence of desired signal in the correlation matrix $R_{i+n}$ is also dealt with.

The paper is organized as follows. In Section 2, Problem formulation and general model is presented. In Section 3 Adaptive beamforming with various beamforming methods are presented along with the Novel Hybrid algorithm -Adaptive colored diagonal loading. In Section 4 simulation experiments are presented. Section 5 contains Results and discussions. Section 6 presents the conclusions.

\section{PROBLEM FORMULATION GENERAL MODEL}

An uniform linear array (ULA) of $\mathrm{M}$ elements or sensors is considered. Let a desired signal $\widetilde{S}_{0}$ from a point source from a known direction $\theta_{0}$ with steering vector ' $a_{0}$ ' and $\mathrm{L}$ number of $\mathrm{J}$ (jammer or) interference signals from unknown directions $\left[\theta_{1}, \theta_{2}, \theta_{3} \ldots \ldots . \theta_{L}\right]$ specified by the steering vectors $\left[a_{1}, a_{2}, a_{3}, \ldots . . a_{L}\right]$, respectively impinge on the array. The white or sensor or thermal noise is considered as ' $n$ '.

A single carrier modulated signal $\widetilde{S}_{0}(t)$ is given by

$$
\widetilde{S}_{0}(t)=S_{0}(t) \cos (2 \pi F c) t
$$

It is arriving from an angle $\theta_{0}$ and is received by the $\mathrm{i}^{\text {th }}$ sensor. The signal $S_{0}(t)$ is a baseband signal having deterministic amplitude and random uniformly distributed phase and $F_{c}$ is the carrier frequency. The symbol $\sim$ is used to indicate that the signal is a pass band signal. Let $\mathrm{X}_{1}(\mathrm{k})$ be the single observation or measurement of this signal made at time instant $\mathrm{k}$, at sensor 1 , which is given as

$$
\begin{gathered}
\mathrm{X}_{1}(\mathrm{k})=\mathrm{a}_{0} \mathrm{~S}_{0}(\mathrm{k})+\left[\mathrm{a}_{1}, \mathrm{a}_{2} \ldots \ldots \mathrm{a}_{\mathrm{L}}\right]\left[\mathrm{J}_{1}(\mathrm{k}), \mathrm{J}_{2}(\mathrm{k}) \ldots \mathrm{J}_{\mathrm{L}}(\mathrm{k})\right]^{\mathrm{T}}+\mathrm{n}(\mathrm{k}) \\
=a_{0} S_{0}(k)+\sum_{j=1}^{L} a_{j} J_{j} *(k)+n(k)
\end{gathered}
$$


Hence the single observation or measurement made at the array of elements at the time instant $\mathrm{k}$, called array snapshot is given as a vector with ' $T$ ' as the transpose,

$$
X(k)=\left[X_{1}(k) X_{2}(k) X_{3}(k) \ldots \ldots X_{M}(k)\right]^{T}
$$

The general model of the steering vector [13] is given as

$$
a_{\theta}=\frac{\left[1 e^{-j\left(\frac{2 \pi}{\lambda} d\right) \cos (\theta)} e^{-j 2\left(\frac{2 \pi d}{\lambda}\right) \cos (\theta)} \ldots \ldots e^{-j(M-1)\left(\frac{2 \pi d}{\lambda}\right) \cos (\theta)}\right]}{\sqrt{M}}
$$

Also it is assumed that the desired signal, interference signals and noise are mutually uncorrelated.

\section{ADAPTIVE BEAMFORMING}

In optimum beamformer, a priori knowledge of true statistics of the array data is used to determine the correlation matrix which in turn is used to derive the beamformer weight vector. Adaptive Beamforming is a technique in which an array of antennas is exploited to achieve maximum reception in a specified direction by estimating the signal arriving from a desired direction while signals of the same frequency from other directions are rejected [17] - [21]. This is achieved by varying the weights of each of the sensors used in the array. Though the signals emanating from different transmitters occupy the same frequency channel, they still arrive from different directions. This spatial separation is exploited to separate the desired signal from the interfering signals. In adaptive beamforming the optimum weights are iteratively computed using complex algorithms based upon different criteria. For an adaptive beamformer, covariance or correlation matrix must be estimated from unknown statistics of the array snapshots to get the optimum array weights. The optimality criterion is to maximize the signal-to-interferenceplus-noise ratio to increase the visibility of the desired signal at the array output. In this paper it is assumed that the angle of arrival of the desired signal is known.

\subsection{Estimation of Correlation Matrix}

The correlation matrix can be estimated $[6,7,8,9]$ using different methods which would result in different performance and behavior of the algorithm. In block adaptive Sample Matrix Inversion technique, a block of snapshots are used to estimate the ensemble average of $R_{x}$ and is written as [8]

$$
\begin{aligned}
& R_{x}=E\left\{x(k) x^{H}(k)\right\}=\frac{1}{M} \sum_{K=1}^{K} x(k) x^{H}(k) \\
& =M \sigma_{S}^{2} a_{0} a_{0}^{H}+R_{j}+R_{n}
\end{aligned}
$$

where $\mathrm{M}$ is the number of snapshots used and $\mathrm{k}$ is the time index, $\sigma_{s}^{2}$ is the power of the desired signal and $R_{j}$ and $R_{n}$ are the jammer and noise correlation matrices, respectively and $\mathrm{H}$ is the complex conjugate transpose. The interference-plusnoise correlation matrix is the sum of these two matrices

$$
R_{j+n}=R_{j}+R_{n}
$$

Where $R_{n}=R_{n} \sigma_{n}^{2} I$, and $\sigma_{n}^{2}$ is the thermal noise power, $\mathrm{I}$ is the identity matrix. It is assumed that thermal noise is spatially uncorrelated.

\subsection{Conventional Beamformer}

The expectation value at the antenna elements is written as $E[x(t)]=\left[\begin{array}{lll}X_{1}(t) & X_{2}(t) \ldots . . X_{M}(t)\end{array}\right]\left[\begin{array}{lll}X_{1}(t) & X_{2}(t) \ldots . . X_{M}(t)\end{array}\right]^{T}$ with

$R=E\left\{x(t) x(t)^{H}\right\}$. The output signal $y(t)=W^{H} x(t)$. This is the conventional beamformer output signal with beamformer weight $\mathrm{w}$. Maximizing the beamformer output problem will result in $P={ }_{w}^{\operatorname{Max}} \sum\left\{|y|^{2}\right\}={ }_{w}^{\operatorname{Max}}\left(w^{H} R w\right)$.Solving this equation gives $w=\frac{a_{\theta}}{\sqrt{a_{\theta}^{H} a_{\theta}}}$ where $a_{\theta}$ is the steering vector.

\subsection{MVDR Beamforming}

If ' $M$ ' number of sensors are used in a beamformer with spacing between them as $d=\lambda / 2$, at any instant

$$
y(n)=s_{0}(n) \cdot \sum_{k=0}^{M-1} W_{k}^{*} e^{-j k \theta_{0}}
$$

where $\theta_{0}$ is the phase difference from the reference input and ' $\theta$ ' may be written as $\theta=(2 \pi \mathrm{d} / \lambda) \sin \phi=\pi \sin \phi$ where $\phi$ is the angle of incidence. To protect all signals which are received from the wanted direction, a linear constraint may be defined as

$$
\sum_{k=0}^{M-1} W_{k}^{*} e^{-j k \theta_{0}}=w^{H}(n) a\left(\theta_{0}\right)=g
$$

The constraint ' $\mathrm{g}$ ' may be interpreted as gain at the look direction which is to be maintained as constant. A spatial filter that performs this function is called a linearly constraint minimum variance beamformer (LCMV). If the constraint $g=1$ then the signal will be received at look direction with unity gain and the response at the look direction is distortionless. This special case of LCMV beamformer is known as minimum variance distortionless response (MVDR) beamformer. Mathematically, a weight vector ' $w$ ' is to be calculated for this constrained optimization problem.

${ }_{w}^{\min } w^{*} R w$ Subject to $w^{*} a_{0}=1$

Now the optimal weight vector may be written as

$$
w=R_{x}^{-1} a(\theta) / a^{H}(\theta) R_{x}^{-1} a(\theta)
$$

This beamforming method experiences the following drawbacks

1) Computational complexity in the order of $O\left(N^{2}\right)$ to $O\left(N^{3}\right)$. 
2) In the case of large array, low sample support i.e(M $>>\mathrm{k}), R_{x}$ may result in singular matrix or illconditioned.

\subsection{Sample Matrix Inversion (SMI)}

Sample matrix Inversion techniques solve the equation $R_{x} W_{0}=r_{d x}$ directly by substituting the maximum likelihood estimates for the statistical quantities $R_{x}$ and $r_{d x}$ to obtain.

$$
\widetilde{W}=\hat{R}_{x}^{-1} \hat{r} d x
$$

The maximum likelihood estimates of the signal correlation and cross correlation are $\widetilde{R}_{x}=\sum_{k=0}^{M-1} x_{k} x_{K}^{H}$ and $r_{d x}=\sum_{k=0}^{M-1} \hat{d} x_{k}$ When the input signal is stationary the estimates only need to be computed once. However in cases where the signal statistics are time varying the estimates must be continuously updated. In SMI the convergence performance is quantified in terms of number of statistically independent sample outer products that must be computed for the weight vector to be within $3 \mathrm{~dB}$ of the optimum.

\subsection{MVDR-SMI Beamformer with Adaptive Colored Diagonal Loading}

MVDR is an optimal minimum variance distortionless response beamformer. It is also referred as the full rank solution as it uses all ' $\mathrm{M}$ ' adaptive degrees of freedom. It resembles the Wiener filter of the form

$$
W=R^{-1} r
$$

MVDR weight vector can be derived as

$$
\begin{gathered}
w_{M V D R}=\frac{\left[\sigma_{i, n}^{2}\right]^{-1} a_{\theta}}{a_{\theta}^{H}\left[\sigma_{i, n}^{2}\right]^{-1} a_{\theta}} \\
=\mathrm{s} \\
=w \odot a_{\theta}
\end{gathered}
$$

where $\mathrm{s}$ is unit norm i.e $a_{\theta}^{H} a_{\theta}=1$ and $\odot$ is the Hadamard product.

A standard method of estimating the covariance matrix is by constructing the sample covariance matrix

$$
\hat{R}_{i, n}=\frac{1}{k} \sum_{k=1}^{K} x_{i, n}(k) x_{i, n}^{H}(k)
$$

$x_{i, n}^{H}(k)$ is the $\mathrm{k}^{\text {th }}$ training sample and $\mathrm{k}$ is the total number of training samples that are available. The sample covariance matrix $\hat{R}_{i, n}$ is the maximum likelihood estimate of the true covariance matrix $R_{i, n}$. Now the approach is called sample matrix inversion with MVDR beam forming and the weights are calculated as

$$
W_{(M V D R-S M I)}=\frac{\hat{R}_{i, n}^{-1} a \theta}{a_{\theta}^{H} \hat{R}_{i, n}^{-1} a \theta}
$$

The MVDR method may suffer from significant performance degradation when there are even small array steering vector errors. Several approaches for increasing robustness to array steering vector errors have been proposed during the past few decades. Diagonal loading, linearly constrained minimum variance (LCMV) beam forming, quadratically constrained beamforming and second order cone programming (SOCP) are some of them. In this work, adaptive colored diagonal loading is proposed to improve the SINR and to eliminate the steering vector errors.

To overcome the above mentioned drawback no. 2 in section III. $\mathrm{C}$, a small diagonal matrix is added to the covariance matrix. This process is called diagonal loading [15] or white noise stabilization which is useful to provide robustness to adaptive array beamformers against a variety of conditions such as direction-of-arrival mismatch, element position, gain, and or phase mismatch and statistical mismatch due to finite sample support[12],[14],[18]-[21]. Because of the robustness that diagonal loading provides it is always desirable to find ways to add diagonal loading to beamforming algorithms. But little analytical information is available in the technical literature regarding diagonal loading [11].

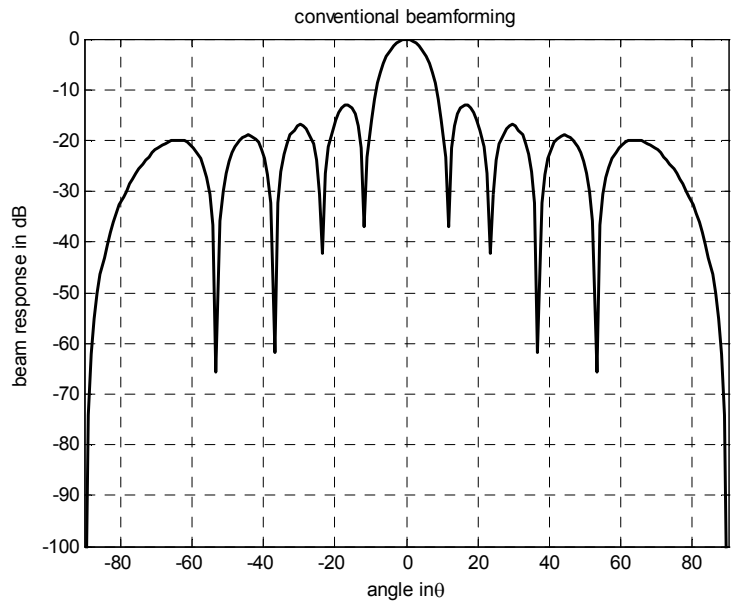

Fig 1 Conventional Beamforming Showing the Beampattern

To achieve a desired sidelobe level in MVDR-SMI beamformer sufficient sample support ' $k$ ' must be available. However due to non-stationarity of the interference only low sample support is available to train the adaptive beamformer. We know that the beam response of an optimal beamformer can be written in terms of its eigen values and eigen vectors. The eigen values are random variables that vary according to the sample support ' $\mathrm{k}$ '. 
Hence the beam response suffers as the eigen values vary. This results in higher sidelobe level in adaptive beam pattern. A means of reducing the variation of the eigen values is to add a weighted identity matrix to the sample correlation matrix.

The result of diagonal loading of the correlation matrix is to add the loading level to all the eigen values. This in turn produces the bias in these eigen values in order to reduce their variation which in turn produces side bias in the adaptive weights that reduces the output SINR. Recommended loading levels of $\sigma_{n}^{2} \leq \sigma_{L}^{2}<10 \sigma_{n}^{2}$ where $\sigma_{n}^{2}$ is the noise power and $\sigma_{L}^{2}$ is the diagonal loading level. The minimum loading level must be equal to noise power. Diagonal loading increases the variance of the artificial white noise by an amount $\sigma_{L}^{2}$. This modification forces the beamformer to put more effort in suppressing white noise rather than interference. When the SOI steering vector is mismatched, the SOI is attenuated as one type of interference as the beamformer puts less effort in suppressing the interferences and noise [17]. However when $\sigma_{L}^{2}$ is too large, the beamformer fails to suppress strong interference because it puts more effort to suppress the white noise. Hence, there is a tradeoff between reducing signal cancellation and effectively suppressing interference. For that reason, it is not clear how to choose a good diagonal loading factor $\sigma_{L}^{2}$ in the traditional MVDR beamformer.

This conventional diagonal loading can be thought of as a gradual morphing between two different behavior, a fully adaptive MVDR solution ( $\mathrm{L}=0$, no loading) and a conventional uniformly weighted beampattern ( $\mathrm{L}=\infty$, infinite loading) [5]. The conventional DL weight vector can be calculated as

$$
W_{M V D R-D L}=\alpha_{M V D R-D L}\left[\hat{R}+\sigma_{L}^{2} I\right]^{-1} a(\theta)
$$

where $\alpha_{M V D R-D L}$ is the normalization constant given by

$$
\alpha_{M V D R-D L}=a(\theta)^{H}\left[\hat{R}+\sigma_{L}^{2} I\right]^{-1} a(\theta)
$$

and $\sigma_{L}^{2}$ reduces the sensitivity of the beampattern to unknown uncertainties and interference sources at the expenses of slight beam broadening [3]. The choice of loading can be determined from L-Curve approach [12] or adaptive diagonal loading.

In the presence of colored noise, DL can be applied which is termed as colored diagonal loading (CDL) and the morphing process may result in a beampattern of our choosing. The colored diagonal loading is similar to $W_{M V D R-D L}$ but the diagonal loading level of $\sigma_{L}^{2}=\infty$, end point, can be altered by the term [5]

$$
W_{M V D R-C D L}=\alpha_{M V D R-D L}\left[\hat{R}+\sigma_{L}^{2} R_{d q}\right]^{-1} a(\theta)
$$

where $R_{d q}$ is the covariance matrix that captures the desired quiescent structure. It may be determined directly based on 1) a priori information - where $R_{d q}$, need not be a diagonal or 2) desired weight vector - where $R_{d q}$ must be diagonal. It is given as

$$
R_{d q}=\operatorname{diag}\left(\left[\operatorname{diag}\left(w_{d q}\right)\right]^{-1} a(\theta)\right)
$$

where $w_{d q}$ is the desired quiescent weight vector.

In Adaptive Colored Diagonal Loading the loading level is calculated assuming the a priori information about the Signal to Noise Ratio (SNR) is available.

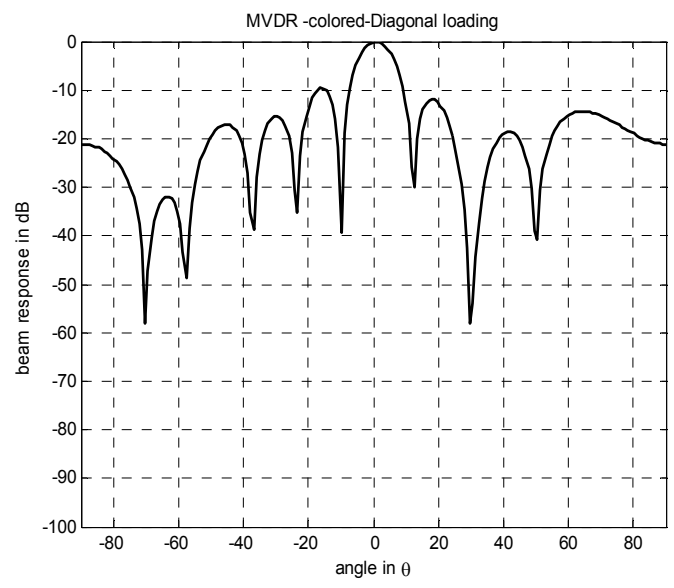

Fig 2 Mvdr-Colored Diagonal Loading

The SNR can be estimated from link budget or using some SNR estimation algorithm. A variable loading MVDR.(VL-MVDR) is proposed in [16] in which the loading level is chosen as $\left(\sigma^{2} \hat{R}\right)$

$$
W_{M V D R-A D L}=\alpha_{M V D R-D L}\left[\hat{R}+\sigma_{A D L}^{2} I\right]^{-1} a(\theta)
$$

where $\sigma_{A D L}=M \cdot S N R$ [4]

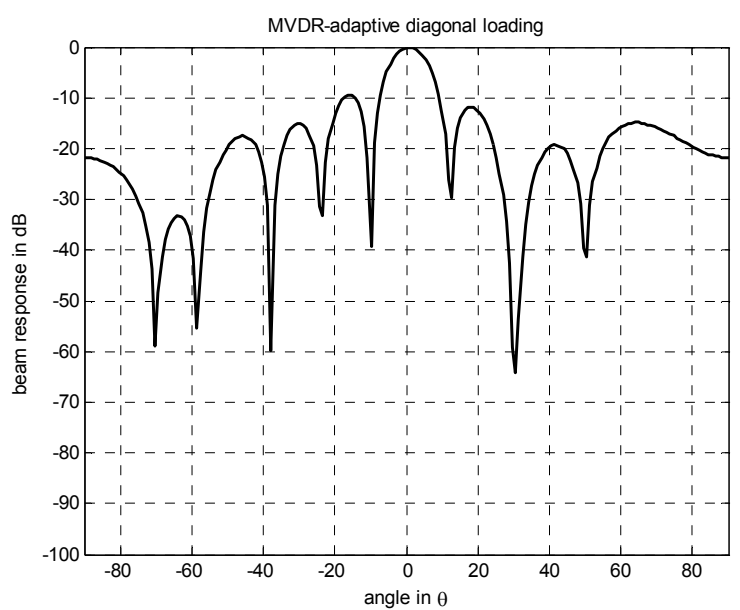

Fig 3 MVDR- Adaptive Diagonal Loading Beampattern 
As already discussed, white noise stabilization is nothing but diagonal loading in which the adaptive colored loading technique is embedded to get a novel hybrid method which is proposed as

$$
W_{M V D R-A C D L}=\alpha_{M V D R-D L}\left[\hat{R}+R_{d q}\right]^{-1} a(\theta)
$$

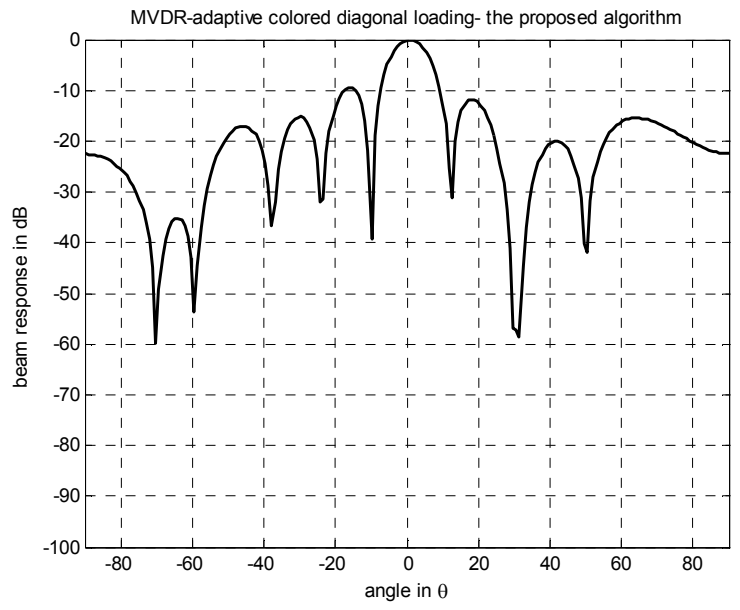
Fig 4 MVDR- Adaptive Colored Diagonal Loading
Beampattern

\section{SIMULATION EXPERIMENTS}

For the proposed hybrid algorithm, a 10 element Uniform Linear Array is considered with SNR of $20 \mathrm{~dB}$ for the desired signal coming from $\theta \mathrm{s}=0^{\circ}$ and INR of $70 \mathrm{~dB}$ for two jammer signals coming from the directions $\theta \mathrm{i}=-70^{\circ}$, and $30^{\circ}$. The element spacing is $d=0.5 \lambda$. The beam patterns for various methods of beamforming are obtained and compared with the performance of MVDR-Adaptive colored Diagonal Loading. Fig 1 shows conventional beamformer. It is observed that the conventional beamformer performs well to get the maximum gain in the desired look direction of $0^{\circ}$. But its performance is worst regarding the cancellation of interferences.

Fig 2 shows the MVDR Colored Diagonal Loading beam pattern which performs much better than the conventional beamformer. This shows a greater improvement in SINR than the conventional. The null is placed properly with out any angle deviation. Fig 3 shows MVDR-ADL beam pattern. Fig 4 shows MVDR-ACDL beam pattern. This beam pattern gives improvement in SINR when compared to other diagonal loading methods. The interferers' angle and their corresponding beam responses are given below.

Interferer 1 at angle $-70^{\circ} \quad:-60 \mathrm{~dB}$

Interferer 2 at angle $30^{\circ}:-60 \mathrm{~dB}$

\section{RESULTS AND DISCUSSION}

\subsection{Number of Elements}

For the ULA which is considered for simulation work, the beampatterns are analyzed by changing the number of elements as $4,8,12,16,24,50$ and 100 .

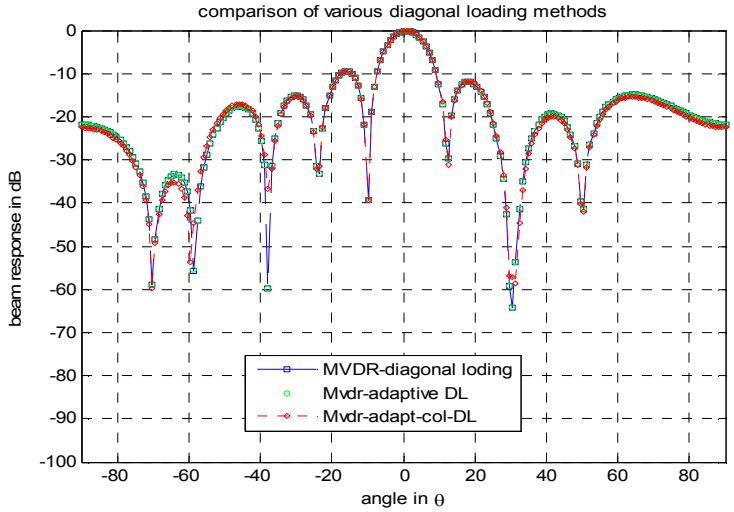

Fig 5 Beampattern of various diagonal loading methods

As the number of elements increases, the beam pattern shows higher resolution i.e the $3 \mathrm{~dB}$ beamwidth becomes much narrower from to $26^{\circ}$ to $1^{\circ}$ for conventional beamformer and $17^{\circ}$ to $1^{\circ}$ for adaptive diagonal loading beamformer. Finer or sharper beams are obtained when more number of elements are used. Sharper the beam, the beamformer is not susceptible to jammers. But the numbers of side lobes are also increased.. A trade off can be obtained to reduce the cost and to have a compact size. Hence a maximum of 16 elements are chosen for further analysis.

\subsection{Noise Effect}

An ULA with 16 elements is considered for analyzing the effect of noise on the peaks of the signal power. Signal to noise ratio (SNR) is varied in steps of $10 \mathrm{~dB}$ starting from $10 \mathrm{~dB}$ till $60 \mathrm{~dB}$. As SNR increases the peak becomes sharper. It shows that the interference sources are suppressed to a maximum extent, so that it will not be a disturbance while extracting the signal even in the presence of strong interferers.

\subsection{Training Issues with the Number of Array Snapshots}

Increasing the number of array snapshots lead to complexity and computational cost but the performance of the beamformer increases. It is a trade off between the cost and the performance. This is shown in Fig 6.

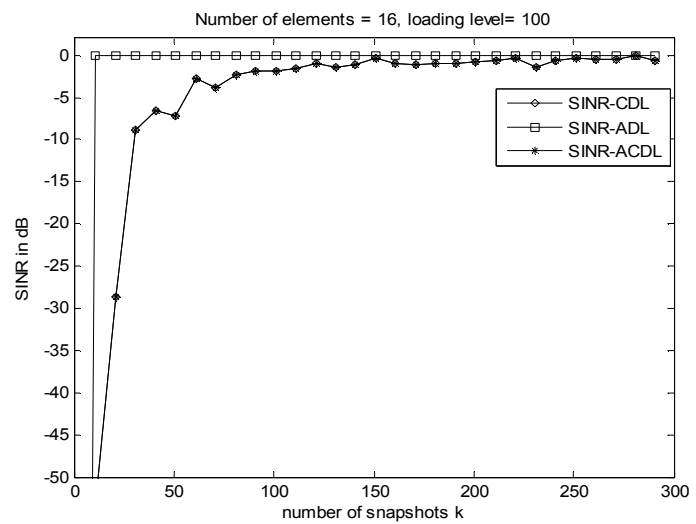

Fig 6 Training issues with the number of snapshots 


\section{CONCLUSION}

In this paper a new Hybrid Robust adaptive beamforming algorithm - MVDR-SMI beamformer with adaptive colored loading is proposed with diagonal loading based on data dependent approach. This method is computationally efficient and proved its effectiveness in improving the SINR over the other methods discussed in this paper.

\section{REFERENCES}

[1] Viktor V.Zaharov, Marvi Teixeira, "SMI- MVDR Beamformer Implementations for Large Antenna Array and Small Sample Size", IEEE Transactions on Circuits and Systems- I Regular Papers Vol.55 No.10, November 2008.

[2] Biao Jiang, Ye Zhu, "A New Robust Quadratic Constraint Beamforming against Array Steering Vector Errors", International Conference on Communications Circiuts and Systems 2004, Vol 2, 27-29 June 2004.

[3] Y.X.Zou,S.C.Chan ,et.al, “ Recursive Robust Variable Loading MVDR Beamforming in Implusive Noise Environment", IEEE Asia Pacific Conference on Circuits and Systems 2008, DOI: 10.1109/APCCAS.2008.4746190

[4] Pekka Lilja, Harri Saarnisaari, "Robust Adaptive Beamforming in Software Defined Radio with Adaptive Diagonal Loading”, IEEE Military Communications Conference 2005, DOI: 10.1109/MILCOM.2005.1606058.

[5] John D. Hiemstra, "Colored Diagonal Loading", Proceedings of the 2002 IEEE Radar conference, DOI: 10.1109/NRC.2002.999682.

[6] H.L.Van Trees, "Detection, Estimation, and Modulation Theory", Part IV, Optimum Array Processing, Wiley, NY, 2002.

[7] Dimitris G.Manolakis, Vinay.K.Ingle, "Statistical and Adaptive Signal Processing”, Artech House, 2005.

[8] Simon Haykin, "Adaptive Filter Theory", Prentice Hall of India, 1996.

[9] Frank Gross, Smart Antennas for Wireless Communications with Matlab, McGraw-Hill, 2005.
[10] Lal.C.Godara, “Applications of Antenna Arrays to Mobile communications, Part I: Performance Improvement, Feasibility, and system considerations", Proceedings of IEEE Vol.85, No.7 July 1997.

[11] Louis B.Fertig, "Statistical Performance of the MVDR Beamformer in the presence of Diagonal Loading" Proc of IEEE Sensor Array and Multichannel Signal Processing Workshop, pp 77-81, 2000

[12] John D. Hiemstra, "Robust Implementations of the Multi stage Wiener Filter", PhD Dissertation, April 4, 2003.

[13] Biao Jiang, Ye Zhu, “A New Robust Quadratic Constraint Beamforming against Array Steering Vector Errors", Proc of International Conference on Communications, Circuits and Systems, 2004, pp 765-768.

[14] Jian Li and Petre Stoica, "Robust Adaptive Beamforming”,John Wiley \& Sons Publications, 2006.

[15] Jian Li and Petre Stoica \& Zwang, "On Robust Capon Beamforming and Diagonal loading" IEEE Trans. signal proc vol 51, pp, 1702-1715 July 2003.

[16] J.Gu and P.J.Wolf, "Robust Adaptive Beamforming using variable loading" In proc IEEE workshop on senior Array and Multichannel signal processing 2006.

[17] Chung-Yang Chen, "Quadratically constrained beamforming Robust against Direction-Of-ArrivalMismatch" IEEE Transactions on signal processing, Vol 55, No.8, Aug 2007.

[18] Arash Khabbazibarmenl, "Robust Adaptive Beamforming Based on Steering Vector Estimation via Semidefined Programming Relaxation", AriXv: 1008.1047vi CS [IT], 5.Aug 2010 .

[19] P.Yang et.al., "Adaptive Beamformer using Interploation Techinque for conformal Antenna Array", Progress in Electromagnetics Research B vol.23,pp 215-228,2010.

[20] Lei Lei, Joni et.al., "Robust Adaptive Beamforming in partly calibrated sparse sensor arrays", IEEE Transactions on Signal Processing, vol 58 No.3.,March 2010.

[21] Ju-Hong et.al., "Robust Adaptive Baemforming for multiple signals of interest on Advanced Frequency Error”,EURASIP Journal on Advanced Signal processing DOI.10.1155/2010/873916,Dec 2010. 